\title{
Subacute transverse myelitis developed depending on lyme disease: a case report
}

\begin{abstract}
Transverse myelitis is a very rare neurologic syndrome caused by inflammation of the spinal cord and occurs in both adults and children. Incidence per year varies from 1-5 per million population. ${ }^{1}$ Lyme borreliosis is a tick-borne anthropozoonosis caused by the spirochete Borrelia burgdorferi. Asymptomatic infections are frequent in Europe, as Borrelia-specific antibodies have been observed in $5-25 \%$ of healthy subjects..$^{2-4}$

The primary stage is characterized by erythema migrans and can be followed by dissemination of disease, which mainly affects the joints, heart or nervous system within weeks to months. ${ }^{5}$ The most frequent neurological manifestation of Lyme borreliosis is meningopolyradiculoneuritis. Neuroborreliosis of the central nervous system (CNS) is rare, and differentiation from other disorders of the CNS with concomitant seropositivity for $B$. burgdorferi can be difficult.

In the literature, a few cases of subacute transverse myelitis (SaTM) with their spinal MRI and cerebrospinal fluid (CSF) findings were reported. ${ }^{6}$ We wanted to discuss a case diagnosed subacute transverse myelitis with MRG and CSF findings.
\end{abstract}

Volume 7 Issue 4 - 2017

\author{
Haluk Gumus \\ Selcuk Univercity, Turkey \\ Correspondence: Selcuk Univercity Alaaddin Keykubat \\ Campus Selcuklu/Konya, Turkey, Tel 9033224I5000, \\ Email dr.halukgumus@gmail.com
}

Received: July 06, 2017 | Published: September 20, 2017

\section{Case presentation}

A 56 years old male patient hospitalized with paresthesias at his legs followed by paraplegia and urinary hesitancy for 3 weeks. We learned that he complained spinal sensibility and crick before 3 weeks and it was followed by paresthesias in both legs in 1-2 days. After 1 week, plegia began in distal right leg subtly, and then it spread in both legs in 2 weeks.

He referred our clinic when he can only ambulated with support, after urinary and fecal hesitancy began with progressive paraplegia in last 2 weeks. There were no fever, dermal lesion, lympadenopathy and organomegaly, in his physical examination. Upper thoracal vertebral spinous processes were sensible at his back side. Muscular strengths were 3-4/5 distally and 4/5 proximally at lower extremity. Also vibration sense was absent at lower extremity. There were hypoesthesia beginning from T5-6 level and pain was increasing with touch. There were increased deep tendon reflexes in lower extremities and bilateral babinski sign and also minimal hypertonia in lower extremities.

Autonomic function examination showed constipation and urinary retention. Cranial and spinal MRG administered to the patient approved with these findings. Blood analyses showed WBC as 14700 , hemoglobin as 14.6 and platelets as 385000 . A lesion extended from T5-6 to T9-10 determined in thoracal MRG (pic.1) Cranial MRG was normal. Lumbar function conducted for transverse myelitis. Lymphocytic pleocytosis (121 leukocytes/ul) determined in CSF analyses and total CSF protein was mildly high $(93 \mathrm{mg} / \mathrm{dl})$. Oligoclonal band and anti-aquaporine were negative. Also rubella and herpes virus were negative. Borrelia burgdorferi IgM level was markedly high $(64 \mathrm{u} / \mathrm{ml})$, thus anti-Borrelia antibodies examined with Western Blood Test and it was positive. Mycoplasma Pneumonia or malignant cells analyses were negative. Antinuclear antibody, antidsDNA, antineutrophil cytoplasmic antibodies, HIV, Treponema, hepatitis B and hepatitis C serology, EBV and VSV were negative also.

Therefore, IV ceftriaxone treatment was given and IV pulse corticosteroid administered for 10 days. Ceftriaxone treatment continued for 7 days and then doxycycline applied for the following 14 days. Subsequently, patient transferred to rehabilitation program.

\section{Discussion}

The term transverse myelitis refers to nonspecific inflammation across one level of the spinal cord. Approximately one third of patients with transverse myelitis report a febrile illness (flu- like with fever) in close temporal relationship to the onset of neurologic symptoms. Transverse myelitis symptoms develop rapidly over several hours to several weeks. Approximately $45 \%$ of patients worsen maximally within 24 hours. Inflammation within the spinal cord interrupts neuronal pathways and causes the common presenting symptoms of transverse myelitis which include limb weakness, sensory disturbance, bowel and bladder dysfunction, and back/radicular pain.

Almost all patients develop leg weakness of varying degrees of severity. Sensation is diminished below the level of spinal cord involvement in the majority of patients. Some experience tingling or numbness in the legs. Pain and temperature sensation are diminished in the majority of patients. Vibration sensation and joint position sense may be decreased or spared. Bladder and bowel sphincter control are disturbed in the majority of patients. Patients sometimes report a tight banding or girdle- like sensation around the trunk that may be very sensitive to touch.

Lyme disease has been reported in many countries, but knowledge about its epidemiologic study in Turkey is incomplete. The southern coast of Anatolia (Mediterranean region) is characterized by the Mediterranean climate, with average temperatures of 9 C in winter and $29 \mathrm{C}$ in summer. Ixodes ricinus, also called the sheep tick or castor bean tick, is seen in this area. ${ }^{7}$ The seroprevalence of $B$. burgdorferi has been found to be as high as $36 \%$ in some rural areas of Turkey, although Lyme disease caused by $B$. burgdorferi is quite rare. ${ }^{8}$

Transverse myelitis may occur in isolation (idiopathic) or in the setting of another illness. It may happen as a parainfectious, paraneoplastic or postvaccinal syndrome or as a complication of systemic autoimmune disease, multiple sclerosis or vasculopathy. ${ }^{9}$ Ropper AH and Poskanzer DC followed 52 patients with acute and 
subacute transverse myelopathy at the Massachusetts General Hospital between 1955 and 1975. Nineteen had symptoms of a recent acute infectious illness, three had cancer, and one had undergone a recent operation had $45 \%$ of their 33 cases mentioned above categorized as parainfectious, $21 \%$ as associated with multiple sclerosis, $12 \%$ as associated with spinal cord ischemia, and $21 \%$ as idiopathic. Parainfectious transverse myelitis may be distinguishable from that associated with multiple sclerosis on the basis of presentation, findings on imaging, and the presence of cerebrospinal fluid oligoclonal bands. Patients with parainfectious transverse myelitis show evidence of spinal cord swelling, whereas patients with multiple sclerosisassociated transverse myelitis have spinal cord plaques on MRI but not swelling. ${ }^{10}$

Oligoclonal bands are absent in patients with parainfectious transverse myelitis as in our case and present in the majority of patients with multiple sclerosis-associated transverse myelitis. In the study of Blanc et al. ${ }^{6}$ Lyme serology was positive in CSF for all three reported cases. In our patient's case, Lyme disease total $\operatorname{IgG}$ and $\operatorname{IgM}$ in the blood was positive $(>1.1 \text { index })^{6}$

In our case, similar to other studies, IgM was found positive in blood and BOS measurement. The diagnostic evaluation for neuroborreliosis should always include a CSF lumbar puncture, leukocyte cell count, and measurement of the B. burgdorferi CSFto-serum antibody index. However, a negative antibody index has been found in up to $30 \%$ of neuroborreliosis cases. In these cases, the typical clinical symptoms, the presence of $B$. burgdorferi IgM and IgG antibodies in the serum, CSF pleocytosis, and a positive response to antibiotic treatment are mandatory criteria for the diagnosis of neuroborreliosis. ${ }^{11}$ The neuropathogenesis of the central nervous system involvement in borreliosis is still poorly understood, and both direct infection and a post infectious immune mediated neural injury are postulated to be involved. The development of neurologic symptoms may be associated with the initial $B$. burgdorferi infection in the central nervous system (peri infectious) or the appearance of neurologic disorders without central nervous system infection (direct toxic or an immune- mediated). ${ }^{12}$

Spinal cord involvement by $B$ burgdorferi is very rare. As a function of geography, LNB would be a rare differential consideration in the evaluation of transverse myelitis. MR imaging findings with LNB myelopathy are characterized by diffuse or multifocal T2weighted cord lesions. In contrast to the classic cervical spinal cord MR imaging abnormalities seen in MS, most patients with LNB do not have macroscopic lesions or magnetization-transfer ratio changes. ${ }^{13}$ Transverse myelitis is generally a one-time occurrence and recuperation generally begins within 1 to 3 months with most patients showing good to fair recovery. ${ }^{14}$ In the Ropper and Poskanzer study, ${ }^{9}$ an acute catastrophic onset was generally associated with back pain and led to a poor outcome in seven and a good outcome in only one of eleven patients. A subacute progressive onset over several days to four weeks (as in our case), generally with ascending paresthesias or leg weakness, was associated with a good outcome in 15 and fair outcome in 17 of 37 patients. The outcome seems to be correlated with the degree of cord enlargement, persistence of increased signal intensity and limited recovery. Atrophy and remaining high signal intensity are noted on late MRI in patients with poor outcome. ${ }^{15}$

In our case, the blood and csf lyme titer was positive and the patient responded quickly to ceftriaxone which was more consistent with transverse myelitis caused by borrelia burgdorferi. IV ceftriaxone and oral doxycycline both were found to be effective, safe, and convenient for treatment of Lyme neuroborreliosis. ${ }^{16}$

\section{Conclusion}

In conclusion, we report the case of a patient with ATM caused by Lyme neuroborreliosis with surprisingly few clinical signsin contrasts to the severe MRI and CSF findings. If there is strong clinical suspicion of Lyme neuroborreliosis-irrespective of a history of a recent tick bite or erythema migrans-appropriate treatment should be started.

\section{Acknowledgments}

None.

\section{Conflicts of interest}

None.

\section{Funding}

None.

\section{References}

1. Lynn DJ, Newton HB, Rae-Grant A. The 5-minute Neurology Consult. Lippincott Williams \& Wilkins, Philadelphia, USA. 2003.

2. Fahrer H, Sauvain MJ, vd Linden S, et al. Prevalence of Lyme borreliosis in a Swiss population at risk (in German). Schweiz Med Wochenschr. 1988;118(2):65-69.

3. Kaiser R, Kern A, Kampa D, et al. Prevalence of antibodies to Borrelia burgdorferi and tick-borne encephalitis virus in an endemic region in southern Germany. Zentralbl Bakteriol. 1997;286(4):534-541.

4. Stanek G, Wormser GP, Gray J, et al. Lyme borreliosis. Lancet. 2012;379(9814):461-473.

5. Steere AC, Coburn J, Glickstein L. The emergence of Lyme disease. $J$ Clin Invest. 2004;113(8):1093-1101.

6. Blanc F, Froelich S, Vuillemet F, et al. Myelite aigue et neuroborreliose [Acute myelitis and Lyme disease. Rev Neurol (Paris). 2007;163(11):1039-1047.

7. Aydin L, Bakirci S. Geographical distribution of ticks in Turkey. Parasitol Res. 2007;101(Supp1 2):S163-S166.

8. Mutlu G, Gultekin M, Ergin C, et al. Investigation of Borrelia burgdorferi antibodies in the Antalya region. Bull Microbiol. 1995;29:1-6.

9. Ropper AH, Poskanzer DC. The prognosis of acute and subacute transverse myelopathy based on early signs and symptoms. Ann Neurol. 1978;4(1):51-59.

10. Jeffery DR, Mandler RN, Davis LE. Transverse myelitis. Retrospective analysis of 33 cases, with differentiation of cases associated with multiple sclerosis and parainfectious events. Arch Neurol. 1993;50(5):532-535.

11. Bennet R, Lindgren V, Zweygberg Wirgart B. Borrelia antibodies in children evaluated for Lyme neuroborreliosis. Infection. 2008;36(5):463-436

12. Lesca G, Deschamps R, Lubetzki C, et al. Acute myelitis in early Borrelia burgdorferi infection. J Neurol. 2002;249(10):1472-1474.

13. Agosta F, Rocca MA, Benedetti B, et al. MR imaging assessment of brain and cervical cord damage in patients with neuroborreliosis. AJNR Am J Neuroradiol. 2006;27(4):892-894.

14. Berman M, Feldman S, Alter M, et al. Acute transverse myelitis: incidence and etiologic considerations. Neurology. 1981;31(8):966-971.

15. Holtås S, Basibüyük N, Fredriksson K. MRI in acute transverse myelopathy. Neuroradiology. 1993;35(3):221-226.

16. Borg R, Dotevall L, Hagberg L, et al. Intravenous ceftriaxone compared with oral doxycycline for the treatment of Lyme neuroborreliosis. Scand J Infect Dis. 2005;37(6-7):449-454. 